\title{
P293
}

\section{Application of Diffracted Waves Analysis to Edge Detection on 3D and 4D Seismic Data}

\author{
F. Alonaizi* (CO2CRC / Curtin University), M. Alshamry (CO2CRC / Curtin \\ University), R. Pevzner (Curtin University), A. Bona (CO2CRC / Curtin \\ University) \& B. Gurevich (CO2CRC / Curtin University / CSIRO)
}

\section{SUMMARY}

Over the past several decades diffracted wave analysis was applied to such problems as detection of small objects on seismic and GPR data, enhanced fault imaging, detection of edges of the geological bodies. Recently we showed using 2D mathematical modelling the potential of diffracted wave analysis for detection of the $\mathrm{CO} 2$ leakage.

In this paper we illustrate application of several diffracted waves imaging techniques based on the phase reversal phenomena for to detection of edges of the objects on 2D data and generalize the approach for 3D acquisition geometry. The proposed technique was tested on physical modelling data and 4D seismic data acquired as a part of CO2CRC Otway project conducted by the Cooperative Research Centre for Greenhouse Gases Technologies (CO2CRC). 


\section{Copenhagen '12}

\section{Introduction}

Over the past several decades diffracted wave analysis was applied to such problems as detection of small objects on seismic and GPR data (Landa et al., 2007), enhanced fault imaging (Vermeulen et. al, 2006), detection of edges of the geological bodies. Recently Alonazi et al (2011) showed using 2D mathematical modelling the potential of diffracted wave analysis for detection of the $\mathrm{CO} 2$ leakage.

To detect sharp edges of the geological bodies one can employ the phase reversal phenomena (Trorey, 1970, Harlan et al., 1983). An example of such phenomena observed on a data from physical modelling is shown in Figure 1. Note that in case of time lapse seismic surveys the area affected by changes at the reservoir level between the consecutive seismic surveys can be considered as such geological body. Thus, application of an imaging technique aiming to detect presence of edge diffraction to the difference volume will give us contours of the area affected by those changes. This can have several important practical applications, such as estimation of the horizontal extent of the $\mathrm{CO}_{2}$ plume in carbon sequestration projects.

In this paper we illustrate application of several imaging techniques based on the phase reversal phenomena to detection of edges of the objects on 2D data and generalize the approach for 3D acquisition geometry. The proposed technique was tested on physical modelling data and 4D seismic data acquired as a part of CO2CRC Otway project conducted by the Cooperative Research Centre for Greenhouse Gases Technologies (CO2CRC).

\section{Diffraction imaging}

Travel time curve of a diffracted wave is given by the equation

$$
t_{i}=\sqrt{t_{\circ}^{2}+\frac{4\left(x_{i}-x_{\circ}\right)^{2}}{v^{2}}} .
$$

In equation (1), $t_{i}$ is the time recorded at a particular horizontal offset $\left(x_{i}-x_{0}\right)$ from the scatterer, and $t_{0}$ corresponds to the zero-offset (vertical) two-way time. The velocity $v$ in equation (1) can be approximated by stacking velocity $\left(v_{N M O}\right)$, which can be assumed known from the velocity analysis of the reflection data.

Kirchhoff time migration of a stacked seismic section is equivalent to summation of samples of the original section along travel time curves given by the equation (1) for every possible position of a scatterer with appropriate weight and followed up the correction of the spectrum. Keydar and Landa (1998) proposed to use semblance function computed in a time window around the travel time curve to produce so-called D-sections:

$$
S=\frac{1}{N} \frac{\sum_{t=-m}^{+m}\left(\sum_{i} f_{i t(i)}\right)^{2}}{\sum_{t=-m}^{+m}\left(\sum_{i} f_{i t(i)}{ }^{2}\right)},
$$

where $f_{i t}$ is the $i^{\text {th }}$ sample at a given time window ( $t$ index), and $N$ is number of channels. Compared to simple summation, the semblance function is more robust with respect to random noise but provides lower vertical resolution of the image.

A typical problem for diffraction imaging is the necessity to suppress specular reflections to recover weak diffracted waves. In case of analysis of the difference volumes generated during time-lapse data analysis, this problem is less severe as majority of the specular reflections is suppressed by the differencing process itself. 


\section{Copenhagen ' 12}

To accommodate phase reversal phenomena observed for the edge diffractions in $2 \mathrm{D}$ data, one can change sign of the signal on one side of the travel time curve. This process maximises the energy of the signal only if there is reverse polarity, otherwise it will cancel it out in case of specular reflections. This approach can be used for both stacking and computation of the semblance.

A possible generalisation of the approach for 3D geometry is presented below. The input data for the analysis is a zero-offset or stacked $3 \mathrm{D}$ volume. This can be either data obtained as a result of single $3 \mathrm{D}$ survey or a difference volume in case of TL seismic survey.

As orientation of a possible edge diffractor in 3D is unknown, we compute a set of D-sections (Dvolumes) corresponding to different orientations of the edges. To do this we divide the full range of possible edge orientations of $\pi$ (due to an obvious symmetry) to angular intervals of $\Delta \alpha$ and flip polarity of the signal for those samples which stay on different semi spaces divided by a vertical plane oriented along correspondent azimuth $n \Delta \alpha$, where $n$ is a sequential number of $\mathrm{D}$-volume.

At the next stage we compute several attributes for every sample of the input volume:

1. Sum of all of the correspondent samples of D-volumes corresponding to a different azimuths of the edges;

2. Maximum values of these samples;

3. Azimuth corresponding to the maximum value of the semblance function.

The first two attributes should give us the position of the edge, the third one - its orientation.

\section{Example 1: Physical modelling data}

To observe phase reversal phenomena we conduct a simple physical lab experiment. We use physical modelling device designed by B.M Hartley at Curtin University (Saul et al., 2007). A block of paper reinforced phenolic was submerged to a water tank. We acquired a constant-offset 2D line along the line oriented perpendicular to the longer edge of the block using a pair of ultrasonic transducers (with central frequency of $\sim 350 \mathrm{kHz}$ ) consisted of 150 shots with $2 \mathrm{~mm}$ spacing between shot locations. Raw data is presented in Figure 1.

We computed three sections: Kirchhoff time migrated, Kirchhoff time migrated with phase reversal for different sides of the travel time curve, and D-section (also taking phase reversal into consideration). The D-section easily delineates the edge of the block showing only the diffracted wave with no reflections as shown in Figure 2.

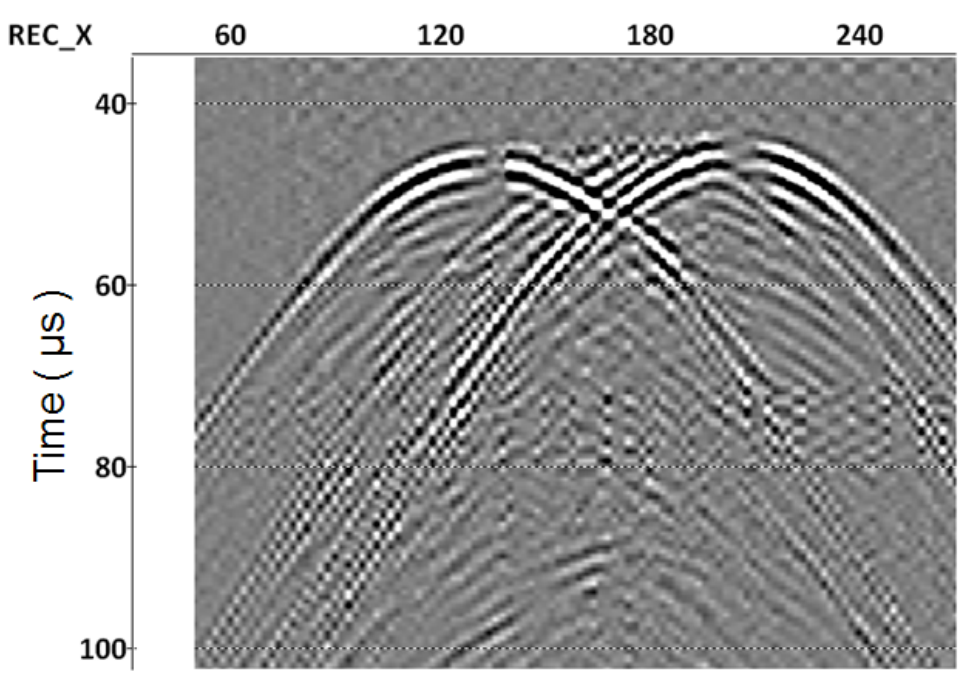

Figure 1 Raw data obtined from the physical mpdel, 2D spatial filter has been applied to show the phase reversal at both edges of the model. 
Copenhagen '12

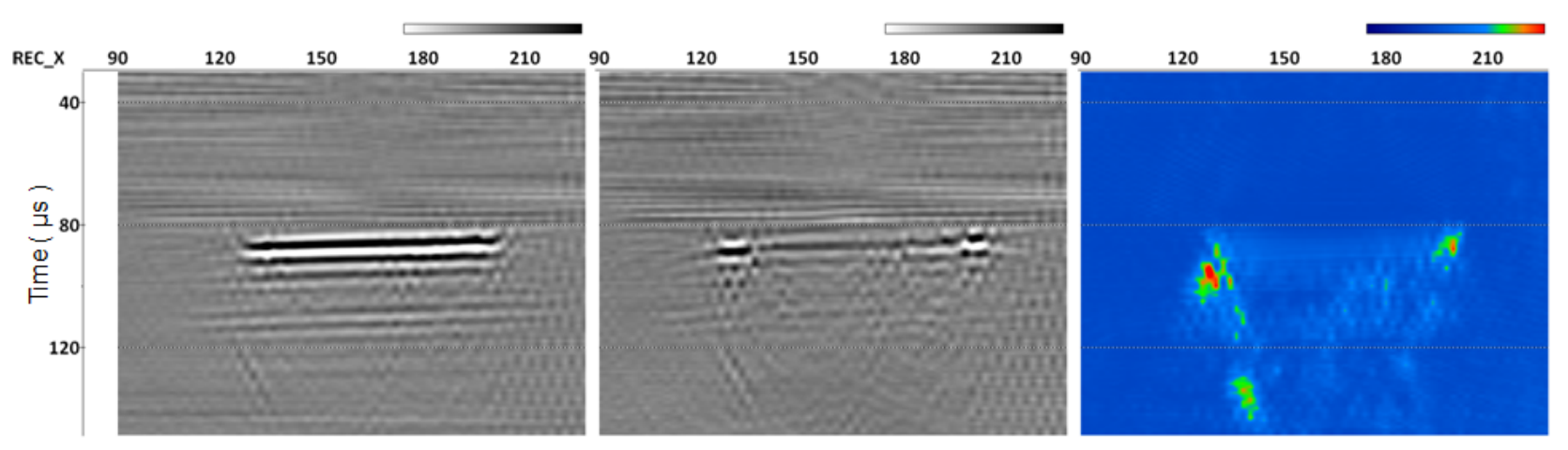

Figure 2 physical model data, (a) after migration, (b) migration with phase reversal, and (c) Dsection of the same data highlighting the edges of the block.

\section{Example 2: CO2CRC Otway project 3D/4D data}

The study area is located in the Victoria's southwest coast of Australia where more than 66,000 tonnes of gas mixture (approximately $80 \%$ of $\mathrm{CO}_{2}$ and $20 \%$ of $\mathrm{CH}_{4}$ ) was injected into onshore depleted gas reservoir (Naylor field, Otway Basin) at around $2 \mathrm{~km}$ depth. The project was conducted by the Cooperative Research Centre for Greenhouse Gas Technologies (CO2CRC) in 2008-2010.

An extensive monitoring and verification program was carried out including several 3D surface seismic surveys. A pre-injection survey was acquired in 2008, and two repeated surveys were acquired in 2009 and 2010, after injection of about 35,000 tonnes and 66,000 tonnes respectively. Another 3D seismic data set acquired in 2000 with much larger areal coverage (which was originally used to discover the Naylor field) was also available for the analysis.

The baseline and monitoring data sets were processed to stacked and migrated volumes using the workflow described by Pevzner et al. (2011) and cross-equalised. The volumes consist of 165 inlines and 190 xlines, bin size is $10 \times 10 \mathrm{~m}$, average fold in the central part is $>100$. Acquisition parameters of the legacy data are very different from the later surveys: area of the $3 \mathrm{D}$ survey is many times bigger, distance between source and receiver lines was $\sim 300 \mathrm{~m}$. This dataset was processed using $20 \times 20 \mathrm{~m}$ bin size, which resulted in a fold of $\sim 30$. The processing flow was similar to the one used for the later surveys. Staked volumes were used for the diffracted wave's analysis. Figure 3 shows an example of an inline 79 of the stacked volume of 2009 survey

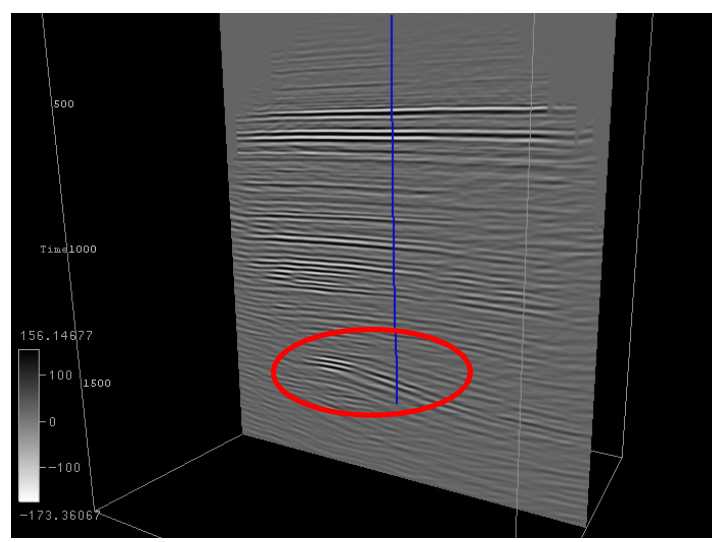

Figure 3 Stack section of inline 79 of the 2009 monitor showing the injection zone at about $1100 \mathrm{~ms}$.

A time slice of a stacked D-volume computed for all azimuths of the edge diffractors for the 2009 survey data is shown in Figure 4 (a). Figure 4 (b) contains minimum similarity attribute computed along the same time slice on a correspondent migrated volume.

As expected, both time slices highlight similar features of the subsurface - fault planes. However the computational approach and the employed phenomena are quite different. 


\section{Copenhagen '12}

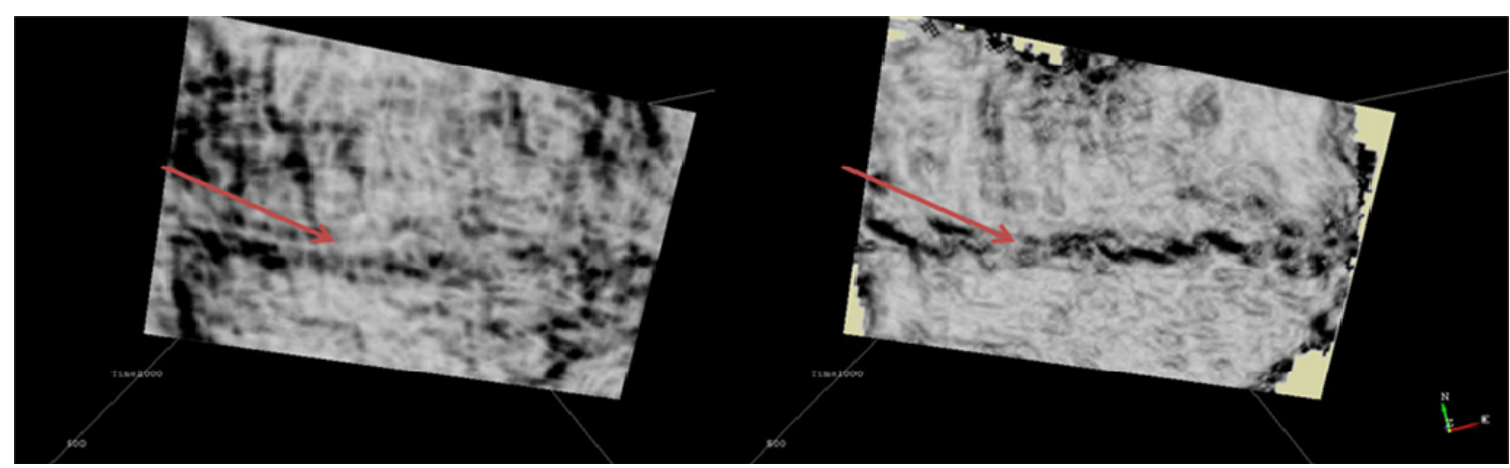

(a)

(b)

Figure 4 A time slice comparison between: (a) D-section and (b) similarity attribute computed for 2009 data. The red arrow shows the fault location agreement in both figures.

\section{Conclusions}

By focusing on diffracted energy, we are able to detect the edge of objects. We generalised this approach from simple 2D physical model to 3D seismic data from Otway project and show the $\mathrm{CO}_{2}$ plume edge. The type of diffraction produced has a phase change of 180 degrees on opposite sides of the diffraction-hyperbola apex. Thus, to avoid amplitude cancellation we considered the signals along each side of the diffraction hyperbola separately. The phase correction maximises the semblance value and improves the detection method. The method can be applied to conventional 2D/3D or TL seismic. Having multiple surveys, we can track the $\mathrm{CO}_{2}$ migration and image its evolution in time. There is agreement between the attribute and $\mathrm{D}$-section that can delineate the fault in the time slice images.

\section{Acknowledgements}

This work is funded by the Commonwealth of Australia through CO2CRC research activities.

\section{References}

Harlan, W.S., Claerbout, J.F. and Rocca, F. [1983] Extracting velocities from diffractions. SEG Technical Program Expanded Abstracts 2(1), 574-577.

Vermeulen, J., Gurevich, B., Urosevic, M. and Landa, E. [2006]. Fault Detection and Mapping Using 3D Diffraction Imaging and Coherency Analysis. 68th EAGE Conference \& Exhibition

Landa, E. and Keydar, S. [1998] Seismic monitoring of diffraction images for detection of local heterogeneities. Geophysics, 63, 1093-1100.

Landa, E., Fomel, A. and Taner, T.M. [2007] Diffraction Imaging for Fracture Detection. EAGE/SEG Research Workshop - Fractured Reservoirs, Integrating Geosciences for Fractured Reservoirs Description.

Saul, M.J., Hartley, B. and Evans, B. [2007] The Virtual Source Method ? Verifying the concept using numerical and physical modeling. ASEG Extended Abstracts 2007(1) $1-6$

Pevzner, R., Shulakova, V., Kepic, A. and Urosevic, M. [2011]. Repeatability analysis of land timelapse seismic data: CO2CRC Otway pilot project case study. Geophysical Prospecting, 59, 6677.

Taner, M.T. and Koehler, F. [1969] Velocity spectra - digital computer derivation applications of velocity functions. Geophysics, 34(6), 859-881.

Trorey, A.W. [1970] A simple theory for seismic diffractions. Geophysics, 35, 762-784. 